\title{
Criminologie
}

\section{Origine et développement du concept de polyvictimisation}

\section{Sherry Hamby, David Finkelhor et Heather Turner}

Volume 47, numéro 1, printemps 2014

La polyvictimisation des jeunes

URI : https://id.erudit.org/iderudit/1024004ar

DOI : https://doi.org/10.7202/1024004ar

Aller au sommaire du numéro

Éditeur(s)

Les Presses de l’Université de Montréal

ISSN

0316-0041 (imprimé)

1492-1367 (numérique)

Découvrir la revue

Citer ce document

Hamby, S., Finkelhor, D. \& Turner, H. (2014). Origine et développement du concept de polyvictimisation. Criminologie, 47(1), 11-15.

https://doi.org/10.7202/1024004ar d'utilisation que vous pouvez consulter en ligne. 


\title{
Origine et développement du concept de polyvictimisation ${ }^{1}$
}

\author{
Sherry Hamby ${ }^{2}$ \\ Professeure \\ Life Paths Research Program and Department of Psychology \\ Sewanee: The University of the South, États-Unis \\ sherry.hamby@sewanee.edu \\ David Finkelhor \\ Directeur \\ Crimes against Children Research Center, University of New Hampshire \\ david.finkelhor@unh.edu \\ Heather Turner \\ Professeure \\ Crimes against Children Research Center, University of New Hampshire \\ heather.turner@unh.edu
}

L'idée de créer le Juvenile Victimization Questionnaire (JVQ), l'outil utilisé pour mesurer la victimisation multiple dans le cadre de la National Survey of Children's Exposure to Violence, est venue lorsque David Finkelhor a réalisé qu'il existait plusieurs instruments de mesure complets dans le domaine de la délinquance, permettant ainsi une évaluation globale de la participation des jeunes à divers actes de délinquance. Pourtant, la recherche relative à la victimisation se trouvait plutôt «en vase clos», c'est-à-dire isolée à l'intérieur de domaines spécialisés. Il existait des questionnaires adaptés pour la violence faite aux enfants, pour l'intimidation, et même pour l'exposition à la violence dans la communauté, mais pas un seul questionnaire pouvant saisir réellement le fardeau total d'exposition à la victimisation d'un enfant.

1. Traduit de l'anglais par Juan Carlos Arellano López.

2. Sewanee: The University of the South, Psychology, 735 University Ave, Sewanee, TN 37383, USA. 
Étonnamment, bien que l'on ait déployé des efforts pour faire le suivi complet des crimes contre les adultes et les adolescents aux États-Unis, incluant ceux qui n'avaient pas été rapportés à la police ou à d'autres autorités, il n'y avait pas de surveillance nationale des crimes commis contre les enfants âgés de moins de 12 ans. Conséquemment, en plus de répondre à un besoin en recherche, le JVQ avait aussi le potentiel de répondre à un besoin important tant sur le plan épidémiologique qu'en matière de politiques publiques.

C'est donc ainsi que l'idée du JVQ est née. Bien que le principe derrière le concept soit simple, le processus de mise au point d'une telle mesure ne l'est pas. L'élaboration d'une mesure complète et globale supposait un examen exhaustif des instruments de mesure existants, non seulement dans un champ d'étude précis, tel le domaine de la violence faite aux enfants, mais à travers un large éventail de sousdisciplines liées à la violence, ayant chacune ses propres méthodologies et conventions de mesure. Plus d'un an a été consacré à une évaluation approfondie de la littérature sur ce sujet, et à l'élaboration préliminaire d'un ensemble de types de victimisation à considérer. L'étape suivante du processus d'élaboration a été de solliciter la rétroaction de nos collègues du Crimes Against Children Research Center et du Family Research Laboratory, travaillant à l'Université du New Hampshire, ainsi que celle de plusieurs autres collègues d'autres institutions.

Cette partie du processus de conception pourrait être qualifiée de phase de «la tour d'ivoire», car elle impliquait principalement des articles de journaux scientifiques et des professeurs. Or, nous étions conscients qu'une mesure efficace devait bien fonctionner avec divers segments de la population et que la compréhension de nombreuses mesures exigeait un niveau d'éducation considérable, voire une formation universitaire. Pour les phases d'élaboration subséquentes, nous avons donc consulté les membres de la communauté, et avons mené neuf groupes focalisés, dont six avec des parents et trois avec des adolescents. Nous leur avons demandé d'évaluer si les concepts définis dans nos questions étaient formulés dans un langage facile à comprendre. Ces groupes ont été une source abondante de commentaires utiles qui ont entraîné de nombreux changements dans le questionnaire. Nous avons ensuite mené 24 entrevues approfondies auprès d'un échantillon de jeunes à haut risque ayant des antécédents de maltraitance documentés. Nous avons effectué un suivi quant à la façon dont ils répondaient aux questions et avons révisé le questionnaire à nouveau afin de nous 
assurer que leur compréhension correspondait à nos concepts des différents types de victimisation.

Suivant cette démarche, que nous croyons être l'un des plus amples processus de mise au point de mesure jamais entrepris dans le domaine de la violence, nous avons obtenu notre premier échantillon représentatif à l'échelle nationale en 2003: le Developmental Victimization Survey (DVS). Cette enquête nous a permis d'établir les propriétés psychométriques du JVQ et de documenter le fardeau réel de la victimisation des jeunes (Finkelhor et al., 2005). Incluant approximativement 2000 participants de 2 à 17 ans, il s'agissait à l'époque d'une enquête relativement vaste sur la victimisation des jeunes pour les États-Unis. Le DVS a mené au premier article ayant officiellement fait référence à la "poly-victimisation", un terme introduit par Heather Turner et David Finkelhor en réalisant que, contrairement à la plupart de stéréotypes, la forme de violence vécue par un enfant importait moins que le nombre de différents types de violence qu'il avait vécus (Finkelhor et al., 2007). La vulnérabilité d'un enfant dans différents milieux de la part d'auteurs multiples et la victimisation sous diverses formes étaient plus importantes que lorsqu'il était la cible d'un seul type de victimisation. Ce principe était même vrai pour les victimisations habituellement considérées comme étant les plus graves: la maltraitance des enfants, l'agression sexuelle et l'exposition à la violence conjugale.

Les conclusions sur la victimisation multiple ont été répliquées en 2008, lorsque nous avons été en mesure d'élargir la DVS à une enquête plus vaste: la National Survey of Children's Exposure to Violence (Finkelhor et al., 2009). Comprenant plus de 4500 jeunes, incluant des nourrissons et des bébés à partir d'un mois (représentés pour l'entrevue par la personne qui en prenait soin), et plus exhaustive dans l'évaluation de certaines formes de victimisation comme l'exposition à la violence familiale, la National Survey of Children's Exposure to Violence (NatSCEV) a fourni une évaluation plus compréhensive de la victimisation des jeunes. Les données obtenues à partir de la NatSCEV ont de nouveau confirmé que la polyvictimisation avait un plus grand impact sur les symptômes traumatiques de cet échantillon représentatif à l'échelle nationale que toutes les formes de victimisation considérées individuellement (Turner et al., 2010). La NatSCEV 2 a été réalisée en 2011 (Finkelhor et al., 2013), et la mise en œuvre de la NatSCEV 3 était prévue vers la fin de l'année 2013. Chaque fois, nous tentons d'enrichir et d'approfondir notre compréhension de la victimisation des 
jeunes. Par exemple, lors de la NatSCEV 2, les mesures sur la négligence envers les enfants ont été améliorées; tandis que la NatSCEV 3 comportera davantage de questions relativement à la violence liée aux armes à feu.

Bien que la polyvictimisation souligne le fardeau réel de la victimisation des jeunes et que les études aient produit des statistiques troublantes, ce programme de recherche rend possibles l'intégration et la synthétisation et l'opportunité d'améliorer les services aux enfants en adoptant une approche holistique centrée sur l'enfant, plutôt qu'une approche fragmentée des problèmes de victimisation des jeunes. Nous croyons qu'il s'agit d'une des raisons principales pour lesquelles les équipes de recherche de nombreux pays et régions ont mené des études sur la polyvictimisation, incluant le Québec (Cyr et al., 2012), le Royaume-Uni (Radford et al., 2013), la Finlande (Ellonen \& Salmi, 2011), l'Espagne (Soler et al., 2012) et la Chine (Chan et al., 2011). Enfin, nous sommes ravis de constater que Katie Cyr et ses collègues fournissent de l'information sur ce nouveau domaine à un public francophone encore plus large et nous espérons qu'une approche davantage axée sur l'enfant à l'égard de la victimisation à l'échelle mondiale permettra éventuellement une meilleure sécurité et un plus grand bienêtre pour tous les enfants.

\section{Références}

Chan, K. L., Brownridge, D. A., Yan, E., Fong, D. Y., \& Tiwari, A. (2011). Child Maltreatment Polyvictimization. Psychology of Violence, 1 (1), 4-15.

Cyr, K., Chamberland, C., Lessard, G., Clément, M.-È., Wemmers, J.-A., CollinVézina, D., Damant, D. (2012). Polyvictimization in a child welfare sample of children and youths. Psychology of Violence, 2 (4), 385-400.

Ellonen, N., \& Salmi, V. (2011). Poly-Victimization as a Life Condition: Correlates of Poly-Victimization among Finnish Children. Journal of Scandinavian Studies in Criminology and Crime Prevention, 12 (1), 20-44.

Finkelhor, D., Hamby, S. L., Ormrod, R. K., \& Turner, H. A. (2005). The Juvenile Victimization Questionnaire: Reliability, validity, and national norms. Child Abuse and Neglect, 29 (4), 383-412.

Finkelhor, D., Ormrod, R. K., \& Turner, H. A. (2007). Poly-victimization: A neglected component in child victimization. Child Abuse and Neglect, 31 (1), $7-26$.

Finkelhor, D., Turner, H. A., Ormrod, R. K., \& Hamby, S. L. (2009). Violence, abuse and crime exposure in a national sample of children and youth. Pediatrics, 124 (5), 1411-1423. 
Finkelhor, D., Turner, H. A., Shattuck, A., \& Hamby, S. L. (2013). Violence, Crime, and Abuse Exposure in a National Sample of Children and YouthAn UpdateNational Violence, Crime, and Abuse Exposure. JAMA pediatrics, 167 (7) 1-8.

Radford, L., Corral, S., Bradley, C., \& Fisher, H. L. (2013). The prevalence and impact of child maltreatment and other types of victimization in the UK: Findings from a population survey of caregivers, children and young people and young adults. Child Abuse $\mathcal{F}$ Neglect, 37 (10), 801-813.

Soler, L., Paretilla, C., Kirchner, T., \& Forns, M. (2012). Effects of polyvictimization on self-esteem and post-traumatic stress symptoms in Spanish adolescents. European child $\mathfrak{\sigma}$ adolescent psychiatry, 21 (11), 645-653.

Turner, H., Finkelhor, D., \& Ormrod, R. (2010). Poly-victimization in a national sample of children and youth. American Journal of Preventive Medicine, 38 (3), 323-330. 